\title{
There is more to trial history than priming in attentional capture experiments
}

\author{
Florian Goller ${ }^{1}$ • Ulrich Ansorge ${ }^{1}$ \\ Published online: 2 April 2015 \\ (C) The Psychonomic Society, Inc. 2015
}

\begin{abstract}
We used contingent attentional capture to investigate whether capture in a given trial $n$ was affected by the cuetarget position relations in a preceding trial $n-1$. Typically, attentional capture by a cue facilitates reaction times for targets in valid conditions (with the cue and target at the same position) relative to invalid conditions (with the cue and target at different positions). Also, this validity effect holds for cues with a feature similar to the searched-for target features (i.e., matching cues), but not for cues dissimilar to the searched-for target features (i.e., nonmatching cues), a pattern termed contingent capture because capture is assumed to be contingent on the match between the cue and top-down control settings. Here, we replicated this contingent-capture pattern with cues that were nonpredictive of the target position. In addition, we showed that during search for white onset targets, red nonmatching color cues also created a validity effect if the same nonmatching cue had been used as a valid cue in trial $n-1$ (Exps. 1 and 2). This intertrial contingency of the nonmatching cue's validity effect was also found if the cues and targets both changed their positions from trial to trial, rendering position priming unlikely (Exp. 2). A similar intertrial contingency was found for nonmatching white onset cues, but not for matching red color cues during search for red color targets (Exp. 3). These results are discussed in light of explanations of the contingent-capture effect and of intertrial contingencies.
\end{abstract}

Florian Goller

florian.goller@univie.ac.at

1 Faculty of Psychology, University of Vienna, Liebiggasse 5, 1010 Vienna, Austria
Keywords Cognitive and attentional control · Attention · Attentional capture

According to contingent attentional capture theory, visual cues attract attention to the degree that they match to the top-down attentional control settings (Folk, Remington, \& Johnston, 1992). Using cues that were not predictive of the target position, Folk et al. found that only top-down matching cues captured attention: A valid cue at the same position as the target facilitated finding the target, as compared to an invalid cue presented away from the target. This validity effect was attributed to the capture of attention by the cue (Posner, 1980). Importantly, the validity effect was absent with cues that did not match the top-down settings. For example, a validity effect of white onset cues was found only if participants searched for white onset targets, but the validity effect was absent if participants searched for red color targets. Folk et al. reasoned that the participants must have set up top-down control settings to search for the target features, and that the cue must have matched these settings to capture attention. In line with this theory, these authors also found the complementary result pattern of a validity effect of red color cues only if participants searched for red color targets, but not if they searched for white onset targets.

An alternative theory proposes that selection history or intertrial priming of attentional capture might contribute to this contingent-capture effect (Awh, Belopolsky, \& Theeuwes, 2012; Theeuwes, 2010; but see Ansorge \& Horstmann, 2007; Ansorge, Kiss, Worschech, \& Eimer, 2009; Eimer \& Kiss, 2008; Folk \& Remington, 2008). For example, attentional capture by a red target in trial $n-1$ might prime capture by a matching red cue in a subsequent trial $n$ by way of cuetarget color similarity (cf. Maljkovic \& Nakayama, 1994). Clearly, a similar priming of attention would not be possible 
with the nonmatching cues, because their color would always differ from that of the target in trial $n-1$.

Here we set out to test a different influence of selection history: the cue-target position relations in trial $n-1$. A number of studies have suggested that attentional capture by a cue (or a distractor) could be stronger after preceding valid trials (or preceding trials without a distractor) than after preceding invalid trials (Jongen \& Smulders, 2007; Mordkoff, Halterman, \& Chen, 2008; Müller, Geyer, Zehetleitner, \& Krummenacher, 2009; Qian, Shinomori, \& Song, 2012). The corresponding intertrial contingency of the validity effect could be due to a number of different processes (Mayr, Awh, \& Laurey, 2003; Schlaghecken \& Martini, 2012; Schmidt, 2013). For example, it could reflect the participants' expectancy of the usefulness of the cue (cf. Gratton, Coles, \& Donchin, 1992). Alternatively, it might also be due to the active suppression of a stimulus that led to conflict (here: of an invalid cue) in $n-1$ (Botvinick, Braver, Barch, Carter, \& Cohen, 2001). Whatever its exact origin, this intertrial contingency has never been studied in contingent-capture experiments. The present study closes this gap.

\section{Experiment 1}

We were interested in the effect of the cue-target position relations in $n-1$ on the validity effects from average nonpredictive cues in trial $n$. These intertrial contingencies of the validity effect were assessed for matching and nonmatching cues. For example, intertrial contingencies of validity effects could be characteristic of all forms of cueing, such as cueing by both matching and nonmatching cues. Alternatively, some forms of cueing, such as by nonmatching cues, might be particularly prone to intertrial contingencies. These were the questions that we addressed using search for onset targets (see Folk et al., 1992).

\section{Method}

Participants A total of 28 participants (19 females, nine males; $M_{\text {Age }}=22.93$ years, $S D_{\text {Age }}=3.22$ ) were recruited from among the psychology students of the University of Vienna. Here and in all following experiments, participants received partial course credit, had normal or corrected visual acuity and normal color vision. Prior to the experiments, informed consent was obtained from all participants. In Experiment 1, one participant was excluded because of an error rate (ER) exceeding $20 \%$. The ERs of the remaining sample ranged between $1.43 \%$ and $15.89 \%$.

Apparatus Stimuli were presented on a 19-in. LCD Monitor (Acer B 193) with an aspect ratio of 4:3 and a resolution of 1 , $024 \times 768$ pixels at a vertical refresh rate of $75 \mathrm{~Hz}$. The viewing distance was kept stable at $57 \mathrm{~cm}$ by a chin-andforehead rest. Manual responses were recorded as keypresses of the left and right index fingers on a keyboard. The experiment was programmed and controlled using MATLAB 7.7.0 (The MathWorks Inc., Massachusetts, USA) and the Psychophysics Toolbox (Brainard, 1997).

Stimuli All stimuli were presented against a black (CIE Lab: $0.8 \mathrm{~cd} / \mathrm{m}^{2} ; 0.1 /-0.1$ ) background. Four gray (CIE Lab: $75.5 \mathrm{~cd} /$ $\left.\mathrm{m}^{2} ;-5.3 /-18.8\right)$ square-shaped outline placeholders $\left(1.5^{\circ} \times\right.$ $\left.1.5^{\circ}\right)$ were presented at the corners of an imaginary diamond around a gray central fixation point $\left(0.5^{\circ}\right)$. The center-to-center distance between the fixation point and the placeholders was 5.0 $0^{\circ}$. On each trial, a white (CIE Lab: $137.7 \mathrm{~cd} / \mathrm{m}^{2} ;-7.8 /-28.4$ ) onset target was shown at the center of one of the placeholders. This target could equally likely be an " $\mathrm{X}$ " or an " $=$ " $\left(1.33^{\circ} \times\right.$ $1.33^{\circ}$ ). The cues were realized as four filled dots (diameter: $0.25^{\circ}$ ) centered on the placeholders, with the dots being $0.13^{\circ}$ away from the placeholders' edges. In the case of the color cues, these dots were red (CIE Lab: $73.9 \mathrm{~cd} / \mathrm{m}^{2} ; 73.9 / 98.7$ ) for one placeholder and white for the remaining three placeholders. In the case of the onset cues, the dots appeared in white color around one of the four placeholders only.

Procedure Figure 1 illustrates the sequence of events in a trial. After fixation $(600 \mathrm{~ms})$, the cueing display was presented for $50 \mathrm{~ms}$, followed by another fixation display for $100 \mathrm{~ms}$. Next, the target display was shown for $50 \mathrm{~ms}$. After the offset of the target, a blank screen was presented until participants responded or the timeout was reached (see below). The stimulus-response mappings were kept constant for each participant but counterbalanced across participants. Reaction times (RTs) were measured to the nearest millisecond as the time intervals between target onset and a keypress. If no keypress was registered within 1,550 ms, participants received an onscreen instruction to react faster, and the trial was counted as an error. In total, the experiment consisted of a minimum of 1 , 536 trials, but since error trials were repeated at the end of the experiment, the exact duration of the experiment varied slightly across participants.

Half of all cues matched the search settings for the target feature (i.e., the white onset cues), whereas the other half did not (i.e., the red color cues). From trial to trial, the cue positions, target positions, and target identities varied randomly, but across trials, both cues and targets were counterbalanced across all four locations. The cue and target positions were $25 \%$ valid, $75 \%$ invalid, and uncorrelated across trials. Invalid cues appeared randomly but equally often at each of three positions away from the target. Cues were also not informative about the target's identity. In addition, we presented each of the two possible targets equally often. Every 384 trials ( $25 \%$ of all trials), there was a self-paced break. Before the start of the experiment, participants received 20 practice trials 

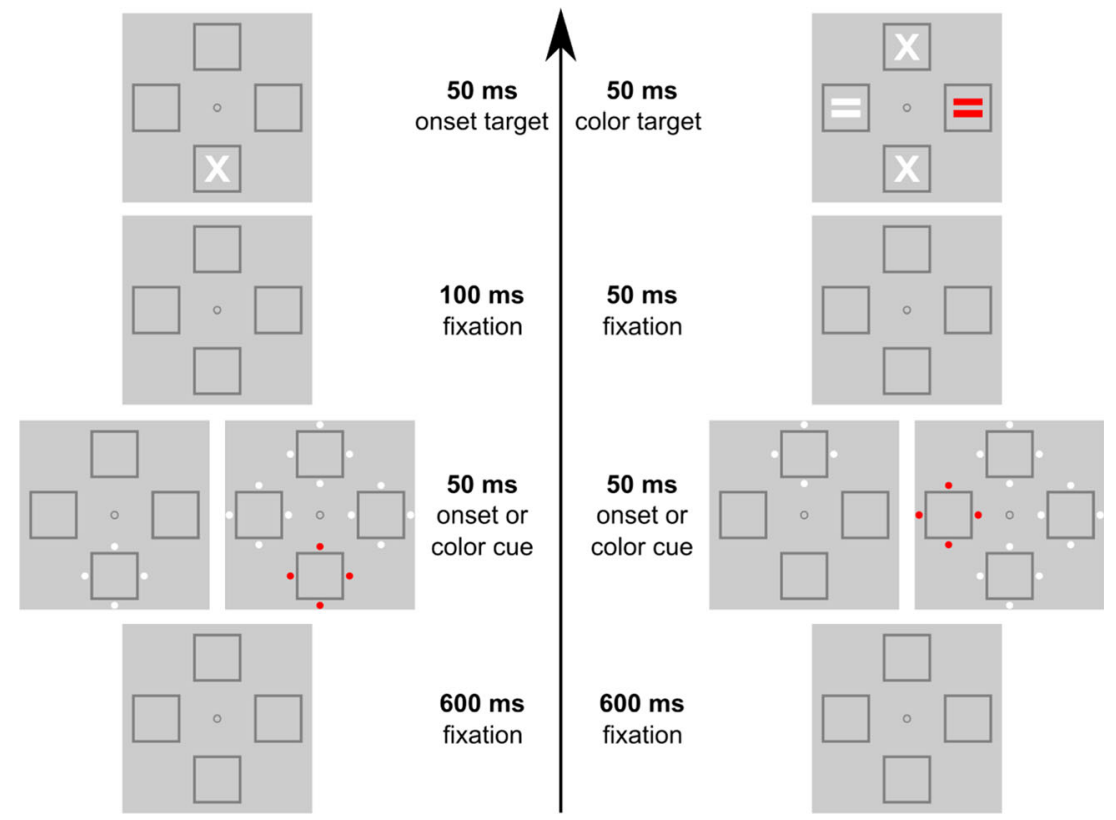

Fig. 1 Example sequences of Experiments 1 and 2 (left side) and Experiment 3 (right side). In each experiment, both color and onset cues were used, whereas the target was of a constant feature throughout

that were chosen randomly from the pool of experimental trials. During practice, participants received on-screen feedback about their answer.

Unlike in Folk et al. (1992), participants only searched for an onset target; there was no second block with a color target. The search for a color target was realized in a separate experiment with different participants (see Exp. 3).

\section{Results}

Reaction times We based the analysis of correct RTs on trials following a correct trial to ensure that participants had applied target-search settings in $n-1$ as intended. Out of all correct trials, $7.51 \%$ that following an error were excluded. Furthermore, RTs below $150 \mathrm{~ms}$ were excluded (an additional $0.04 \%$ ). We computed validity effects as the mean correct RTs in invalid minus the mean correct RTs in valid trials, separately for each participant and condition. Unless otherwise stated, all validity effects (or their reversals; see below) are significantly different from zero (all $p \mathrm{~s}<.05$ ). All results in this article are reported in this way. Validity effects were subjected to a repeated measures analysis of variance (ANOVA), with $n-1$ cue type ( $n-1$ matching, $n-1$ nonmatching), $n-1$ validity ( $n-1$ valid, $n-1$ invalid), and $n$ cue type ( $n$-matching, $n--$ nonmatching) as within-subjects variables. Table 1 (top rows) provides a detailed overview of the RTs and ERs of Experiment 1.

The validity effect was stronger following an $n-1$ valid trial (23 ms) than following an $n-1$ invalid trial $(11 \mathrm{~ms})$, indicated by a main effect of $n-1$ validity, $F(1,26)=10.61, p<.01$. A

the experiment (onset targets in Exps. 1 and 2, and color targets in Exp. 3). The figure is not drawn to scale and in both experiments, a black background color was used

main effect of $n$ cue type, $F(1,26)=105.24, p<.01$, reflected a validity effect that was only present with $n$-matching cues (36 ms), but absent with $n$-nonmatching cues [ $-2 \mathrm{~ms} ; t$ test against zero: $t(26)=-1.02, p=.32]$. These results correspond to the classic finding of contingent capture. Furthermore, we found an interaction between $n-1$ validity and $n$ cue type, $F(1$, 26) $=11.77, p<.01$, which is best considered in light of the also-significant interaction between all variables, $F(1,26)=$ $6.15, p=.02$, which we will discuss next (see also Fig. 2). All other effects did not reach statistical significance, all nonsignificant $F \mathrm{~s}<2.30$, all $p \underline{\mathrm{s}}>.14$.

We conducted separate follow-up ANOVAs split up for the levels of $n$ cue type to analyze more closely how $n$ matching and $n$-nonmatching trials were influenced by $n-1$ cue validity and $n-1$ cue type. In $n$-matching trials, we found no evidence of an intertrial contingency, but only an interaction between $n-1$ cue type and $n-1$ validity, $F(1$, $26)=4.23, p=.05$. Post-hoc comparison of the validity effects after an $n-1$ valid versus an $n-1$ invalid trial (separately for the levels of $n-1$ matching) revealed no significant differences (all $p$ s $>.20$ ). No other effects were found in the follow-up analysis of the $n$-matching trials, all nonsignificant $F_{\mathrm{s}}<0.44$, all $p \mathrm{~s}>.51$.

Looking at $n$-nonmatching trials, we found an effect of $n-1$ validity, $F(1,26)=31.46, p<.01$, reflecting an intertrial contingency: The validity effect was present following $n-1$ valid trials $(10 \mathrm{~ms})$ but was reversed following $n-1$ invalid trials $(-13 \mathrm{~ms})$. No other effects were found in the follow-up analysis of the $n$-nonmatching trials, all nonsignificant $F_{\mathrm{s}}<$ 1.31 , all $p$ s $>.26$. 
Table 1 Reaction times (RTs; in milliseconds) and error rates (ERs; in \%) as a function of cue type (matching vs. nonmatching) and cue validity (valid vs. invalid) for both trials $n$ and $n-1$ in Experiment 1 (upper rows), Experiment 2 (middle rows), and Experiment 3 (lower rows)

\begin{tabular}{|c|c|c|c|c|c|c|c|c|c|c|}
\hline & \multirow[b]{4}{*}{ Trial $n-1$} & & \multicolumn{8}{|c|}{ Trial $n$} \\
\hline & & & \multicolumn{4}{|c|}{ Matching Cue } & \multicolumn{4}{|c|}{ Nonmatching Cue } \\
\hline & & & \multicolumn{2}{|c|}{ Valid } & \multicolumn{2}{|c|}{ Invalid } & \multicolumn{2}{|c|}{ Valid } & \multicolumn{2}{|c|}{ Invalid } \\
\hline & & & RT & ER & RT & ER & RT & ER & RT & ER \\
\hline \multirow[t]{4}{*}{ Experiment 1} & \multirow[t]{2}{*}{ Matching cue } & Valid & 449 & 7.80 & 491 & 7.41 & 466 & 6.92 & 471 & 7.53 \\
\hline & & Invalid & 451 & 7.46 & 483 & 7.10 & 481 & 6.98 & 467 & 6.54 \\
\hline & \multirow[t]{2}{*}{ Nonmatching cue } & Valid & 454 & 9.66 & 485 & 7.68 & 458 & 6.89 & 472 & 7.56 \\
\hline & & Invalid & 452 & 6.07 & 489 & 6.61 & 480 & 7.86 & 467 & 6.11 \\
\hline \multirow[t]{4}{*}{ Experiment 2} & \multirow[t]{2}{*}{ Matching cue } & Valid & 433 & 5.87 & 470 & 8.01 & 452 & 5.85 & 448 & 6.56 \\
\hline & & Invalid & 435 & 6.48 & 456 & 8.24 & 450 & 6.15 & 437 & 6.93 \\
\hline & \multirow[t]{2}{*}{ Nonmatching cue } & Valid & 440 & 6.35 & 470 & 7.61 & 437 & 5.92 & 457 & 7.62 \\
\hline & & Invalid & 431 & 7.70 & 455 & 8.07 & 450 & 6.81 & 442 & 7.79 \\
\hline \multirow[t]{4}{*}{ Experiment 3} & \multirow[t]{2}{*}{ Matching cue type } & Valid & 450 & 5.45 & 522 & 6.37 & 472 & 5.10 & 482 & 4.11 \\
\hline & & Invalid & 427 & 5.61 & 506 & 7.93 & 479 & 4.26 & 477 & 3.78 \\
\hline & \multirow[t]{2}{*}{ Nonmatching cue } & Valid & 436 & 4.71 & 513 & 8.27 & 462 & 4.33 & 485 & 2.39 \\
\hline & & Invalid & 427 & 5.47 & 501 & 7.93 & 478 & 4.22 & 471 & 3.58 \\
\hline
\end{tabular}

Error rates Validity effects were computed from the arcsinetransformed ERs and analyzed analogously to the RTs. For better transparency, the means of the untransformed ERs are reported. An interaction between $n-1$ validity and $n$ cue type, $F(1,26)=7.57, p=.01$, showed that for $n$-matching trials, the validity effects following an $n-1$ valid trial (1.55\%) and following an $n-1$ invalid trial $(0.73 \%)$ were not different from zero (both $p \mathrm{~s}>$.28). For $n$-nonmatching trials, we found a strong trend toward a validity effect following an $n-1$ valid trial [2.01\%; $t$ test against zero: $t(26)=1.98, p=.06]$, whereas

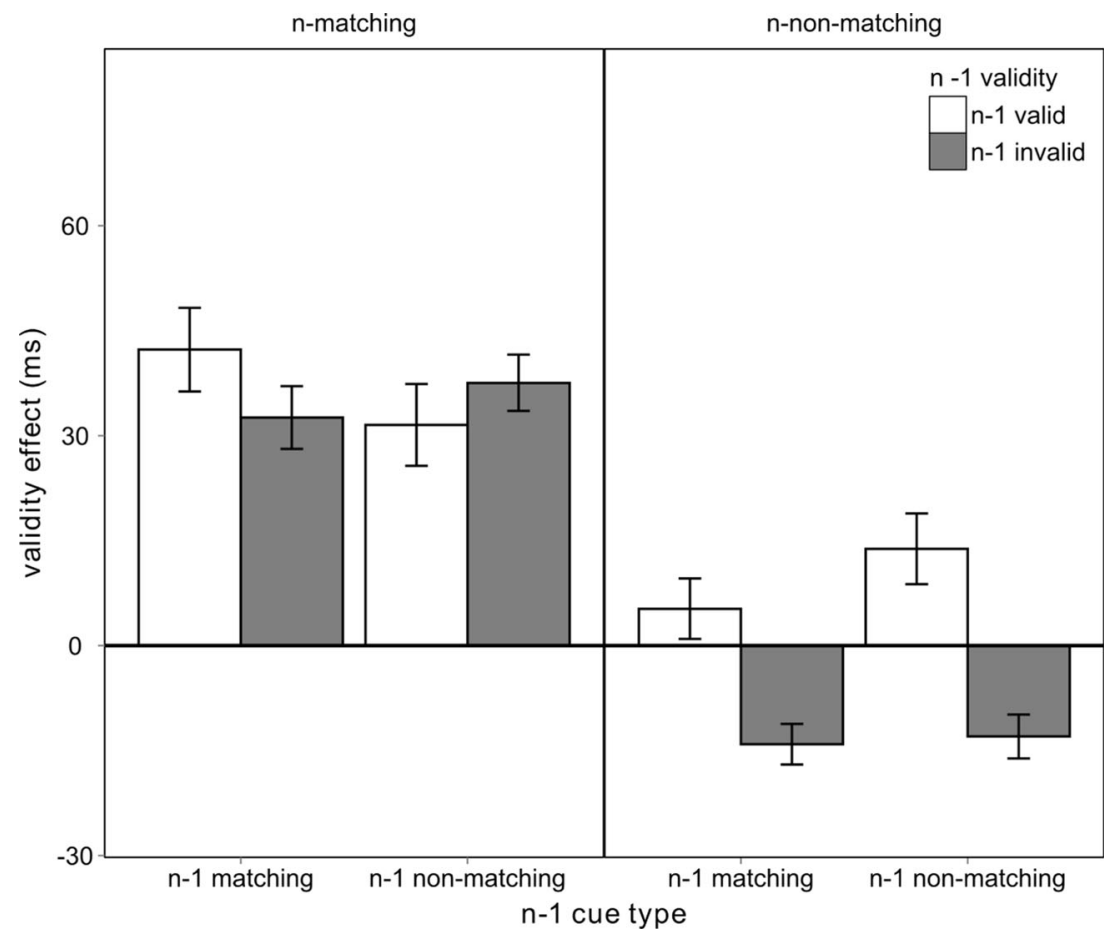

Fig. 2 Validity effects ( $n$-invalid RT $-n$-valid RT) as a function of $n$ cue type ( $n$-matching, left panel; $n$-nonmatching, right panel), $n-1$ validity $(n-1$ valid, white bars; $n-1$ invalid, gray bars), and $n-1$ cue type ( $x$-axis) in Experiment 1 (onset search). Error bars represent the standard errors of the means (SEMs) 
no such trend was found following $n-1$ invalid trials [ $0.65 \%$; $t$ test against zero: $t(26)=-1.36, p=.18]$. No other significant effects were found, all nonsignificant $F \mathrm{~s}<2.23$, all $p \mathrm{~s}>.15$.

\section{Discussion}

Experiment 1 yielded two main findings. First, a larger validity effect in top-down matching than in nonmatching trials reflected contingent capture. Interestingly, this effect was only present on average. A closer look revealed that in the $n$ nonmatching conditions, the null effect was due to averaging across standard and inverted validity effects. Second, a larger validity effect following $n-1$ valid than $n-1$ invalid trials corresponded to an intertrial contingency of the validity effect. Follow-up analyses of the four-way interaction between all variables demonstrated that the intertrial contingency was restricted to the $n$-nonmatching conditions. Here, we only found a validity effect following $n-1$ valid trials, whereas this effect reversed after $n-1$ invalid trials. For the $n$-matching conditions, we found a tendency toward such an intertrial contingency, but no significant pairwise comparisons. Furthermore, in the ERs, we found a trend toward a validity effect after $n-1$ valid trials, but only in the $n$-nonmatching condition. No such trend was found in the $n$-matching condition. In addition to the validity effects in RTs, this result might be regarded as further weak evidence for intertrial contingencies of nonmatching cues.

These results show that more evidence for attentional capture can be found in nonmatching conditions when one scrutinizes the influence of the trial history with respect to factors beyond color priming. The influence of more or less conflict between relevant targets and irrelevant cues in trial $n-1$ on validity effects can be attributed to one of several possible sources. For example, according to the influential conflictmonitoring hypothesis of Botvinick et al. (2001; Carter \& van Veen, 2004), participants would try to shield themselves more from irrelevant information that has been harmful to their performance than in a situation in which this information has been helpful. According to this explanation, active suppression of the nonmatching (irrelevant) cue following an invalid trial would imply that a target at the cued position could also be affected by (partial) suppression of this position. As a consequence, the validly cued target on trial $n$ would be processed at slightly higher costs than an invalidly cued target (cf. Anderson \& Folk, 2012; Belopolsky, Schreij, \& Theeuwes, 2010; Carmel \& Lamy, 2014). This is exactly the pattern that we observed for the $n$-nonmatching cues following an $n-1$ invalid trial.

But why was no similar intertrial contingency found with the matching cues? One reason could be that suppression was easier with the nonmatching cues. For example, active deallocation could be one of the means by which conflict is regulated. According to Theeuwes, Atchley, and Kramer
(2000), participants are better at deallocating their attention away from nonmatching cues than from matching cues. Thus, the stronger validity effects with $n$-matching than with $n$-nonmatching cues could be due to a faster and more efficient deallocation of attention with an $n$-nonmatching than with an $n$-matching cue (Theeuwes et al., 2000). In the $n$-matching conditions, too little deallocation could have prevented successful conflict control after an $n-1$ invalid trial.

Alternatively, active suppression could have nothing to do with attentional capture (Carmel \& Lamy, 2014) or might occur in advance of attentional capture (Anderson \& Folk, 2012). According to the latter view, it is also possible that following an $n-1$ invalid trial, participants suppressed the nonmatching cues more efficiently right from the outset of the displays. Targets at the position of the nonmatching cues would therefore have been subject to this attention-preceding suppression in trial $n$.

Another possibility is that participants might have learned to attend to the target and/or to suppress the cue in invalid trials more efficiently (cf. Verguts \& Notebaert, 2008). This contingency-learning theory could also explain why intertrial contingencies were restricted to the $n$-nonmatching conditions: The salient color of the nonmatching cue would be associated only with the irrelevant cue, whereas the single white abrupt onset of the matching cue would also be associated with the target. Consequently, discriminative learning of selectively attending to targets versus cues could have been stronger with $n$-nonmatching than with $n$-matching cues.

\section{Experiment 2}

Experiment 2 was a replication of Experiment 1, except for the intention to rule out position priming (Maljkovic \& Nakayama, 1996; Mayr et al., 2003; see also Hommel, 1998; Hommel, Proctor, \& Vu, 2004, for a related idea) as a factor generating the stronger validity effect of the nonmatching cues following $n-1$ valid conditions. The intertrial contingency of $n$-nonmatching conditions in Experiment 1 could have been based on the exact repetitions of cue positions from trials $n-1$ to $n$. For example, maybe participants encoded the full episode of a trial $n-1$, consisting of the cue and target colors and their locations. Retrieval of this encoded episode in a subsequent trial $n$ could have facilitated target responses when trial $n$ was a full repetition of the episode in $n-1$ (cf. Huang, Holcombe, \& Pashler, 2004). Because facilitation by full repetitions would have occurred only in (1) $n-1$ $\mathrm{valid} / n$ valid and (2) in $n-1$ invalid $/ n$ invalid sequences, full repetitions would have boosted the validity effect (calculated as $n$-invalid RT minus $n$-valid RT) following $n-1$ valid trials, and decreased it following $n-1$ invalid trials. To test this possibility, Experiment 2 included no cue or target position repetitions across consecutive trials. Under these conditions, no 
trial $n$ was a full replication of its immediately preceding trial $n-1$.

Our predictions were straightforward. If an influence, such as the strategic use of knowledge about the cue's utility (Gratton et al., 1992) or conflict adaptation (Müller et al., 2009), accounted for the intertrial contingencies of the validity effect for the nonmatching cues, we expected to replicate the findings of Experiment 1, because the potential influences of these factors were not affected by our manipulation. If, however, position priming was crucial for an increased validity effect with nonmatching cues following $n-1$ valid trials, we expected to see no such intertrial contingency in the present experiment. The fact that cue and target positions were not repeated from trial to trial also implies that more contingency learning of the specific target position in $n-1$ invalid than in $n-$ 1 valid trials could not be responsible for the any intertrial contingencies that we might observe.

\section{Method}

Participants A group of 21 new participants (nine female, 12 male; $M_{\text {Age }}=23.53$ years, $S D_{\text {Age }}=3.37$ ) was tested. Two participants were excluded because they made more than $20 \%$ errors. The ERs of the remaining sample ranged from $0.45 \%$ to $13.22 \%$.

Procedure The procedure was the same as in Experiment 1, with the notable exception that the positions of targets and cues were never repeated in subsequent trials. For example, if the cue and target were presented on the left in an $n-1$ valid trial, an $n$ valid trial could have contained the cue and target on the right, at the bottom, or at the top, but never on the left. Accordingly, in the case of $n-1$ valid trials with the cue and target on the left, an $n$ invalid trial could have contained a cue at the top and a target at the right or the bottom, a cue at the right and a target at the top or the bottom, or a cue at the bottom and a target at the top or the right, but neither the cue nor target would have appeared on the left.

\section{Results}

Reaction times We removed trials following errors $(9.30 \%)$ and with RTs below $150 \mathrm{~ms}$ (additional $0.03 \%$ ). Unless otherwise stated, all validity effects (or their reversals; see below) are significantly different from zero (all $p \mathrm{~s}<.05$ ). Table 1 (middle rows) provides a detailed overview of the RTs and ERs for Experiment 2 (see also Fig. 3). A main effect of $n-1$ validity, $F(1,18)=8.24, p=.01$, reflected an $n$-validity effect following $n-1$ valid trials (21 ms) that was absent following $n-1$ invalid trials [ $6 \mathrm{~ms}$; $t$ test against zero: $t(18)=1.69, p=$ .11]. Furthermore, the validity effect was only different from zero in $n$-matching trials $(28 \mathrm{~ms}$ ) but not in $n$-nonmatching trials [ $-2 \mathrm{~ms}$; $t$ test against zero: $t(18)=-0.43, p=.67$ ], as was indicated by a main effect of $n$ cue type, $F(1,18)=23.75, p<$ .01. A borderline significant main effect of $n-1$ cue type, $F(1$, $18)=3.38, p=.08$, and an interaction between $n-1$ cue type and $n$ cue type, $F(1,18)=7.84, p=.01$, will be resolved together with the analysis of the also-significant interaction between all variables, $F(1,18)=7.09, p=.02$. No other significant effects were found, all nonsignificant $F_{\mathrm{S}}<.94$, all $p \mathrm{~s}>.34$.

The follow-up analysis of the $n$-matching trials revealed no significant effects, all $F_{\mathrm{s}}<2.10$, all $p \mathrm{~s}>.16$.

For $n$-nonmatching trials, we found effects of $n-1$ cue type, $F(1,18)=13.47, p<.01$, and of $n-1$ validity, $F(1,18)=7.98$, $p=.01$. These two significant effects were qualified by their interaction, $F(1,18)=9.53, p<.01$. If the preceding trial had used a nonmatching cue, the validity effect was only present following $n-1$ valid trials (19 ms) but absent following $n-1$ invalid trials [ $-8 \mathrm{~ms}$; $t$ test against zero: $t(18)=-1.50, p=.15$ ]. If $n-1$ had used a matching cue, the validity effect was inverted following $n-1$ invalid trials $(-13 \mathrm{~ms})$ and absent following $n-1$ valid trials [ $-4 \mathrm{~ms} ; t$ test against zero: $t(18)=-$ $0.73, p=.48]$.

Error rates The main effect of $n-1$ validity came closest to significance, $F(1,18)=3.09, p=.10$, with a tendency toward a larger validity effect after $n-1$ valid trials $(2.67 \%)$ than after $n-1$ invalid trials $(0.77 \%)$. No other significant effects were found, all nonsignificant $F \mathrm{~s}<1.82$, all $p \mathrm{~s}>.19$.

\section{Discussion}

The important results of Experiment 2 closely resembled those of Experiment 1. Again, we found that validity in trial $n-1$ had no impact on the validity effect of $n$-matching cues, but only on the validity effect of $n$-nonmatching cues. A validity effect of the $n$-nonmatching cues was only found if the cue in trial $n-1$ had been both valid and nonmatching. In addition, evidence of active suppression of the nonmatching cue (reflected in an inverted validity effect) was found only if the preceding trial had used a matching and invalid cue. If the preceding trial had used a matchingvalid or a nonmatching-invalid cue, there was no validity effect of the nonmatching cue.

These intertrial contingencies of the validity effect in the $n$-nonmatching conditions were found even though the cue and target in trials $n-1$ and $n$ were never presented at the same positions. Due to this manipulation, the number of possible target positions was effectively reduced, meaning that search for the targets was easier and that new forms of predictability of the target (and cue) were possible. However, position priming and contingency learning of specific target positions were ruled out as factors that could be responsible for the intertrial contingency. This fact in turn makes it likely that factors such as expectancy changes or conflict adaptation lowered the 


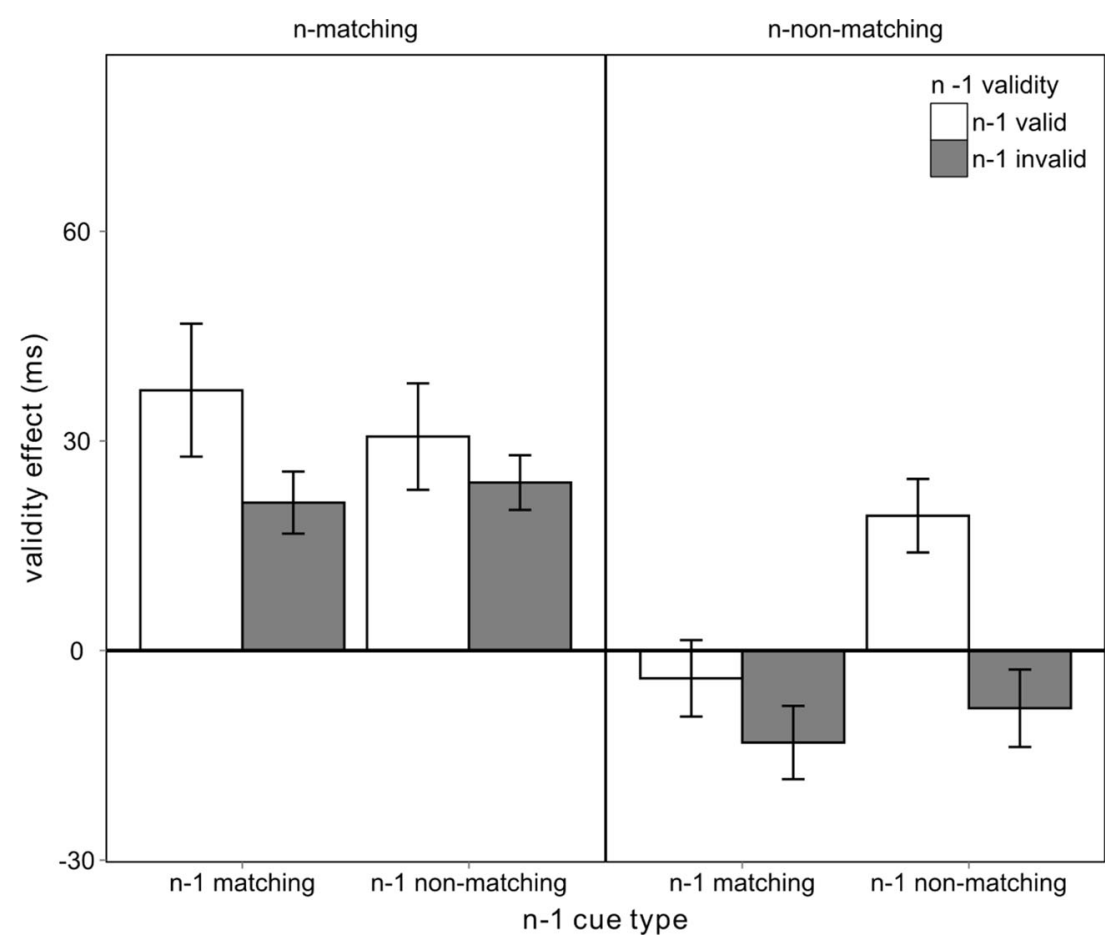

Fig. 3 Validity effects ( $n$-invalid RT $-n$-valid RT) as a function of $n$ cue type (n-matching, left panel; $n$-nonmatching, right panel), $n-1$ validity $(n-1$ valid, white bars; $n-1$ invalid, gray bars), and $n-1$ cue type ( $x$ -

validity effects of $n$-nonmatching cues that were preceded by $n-1$ invalid cues.

However, there were also differences from Experiment 1. In the present experiment, preventing position priming brought to light a cue-color repetition effect in the nonmatching conditions: In Experiment 2, comparing $n-1$ valid versus $n-1$ invalid trials reflected a validity effect versus no validity effect if the nonmatching cue was shown in both trials $n-1$ and $n$, whereas it reflected no validity effect versus an inverted validity effect if a matching cue in $n-1$ preceded a nonmatching cue in trial $n$. This result suggests that attentional capture of nonmatching cues benefited from cue-color repetitions across subsequent trials. This finding would be consistent with an episodic retrieval account, in so far as not only the last attended-to color (that of the $n-1$ target) accounted for a cue's capture in the subsequent trial. The fact that this form of color priming was only found in the present but not the previous experiment might have been due to the cue positions never repeating across trials of Experiment 2, since participants might have focused more on cue colors than on cue positions during the encoding and retrieval of trial episodes. In Experiment 1, cue-position repetitions might have discouraged this kind of color-directed encoding and retrieval strategy.

In addition, several other key findings of Experiment 1 and of the existent literature were replicated. Most importantly, we again found clear evidence for contingent capture, although, as in Experiment 1, it was possible that the selective presence axis) in Experiment 2 (onset search without position repetition between trials $n-1$ and $n$ ). Error bars represent the standard errors of the means $(S E M \mathrm{~s})$

of the validity effect for $n$-matching trials reflected more intertrial priming of cue colors in $n$-matching than in $n$ nonmatching conditions. Crucially, however, as in Experiment 1, intertrial contingency was prevented in the matching conditions, indicating a lower vulnerability of attentional capture in these conditions to the influence of validity in a preceding trial.

\section{Experiment 3}

Here, we tested whether $n-1$ validity affected validity effects when participants searched for a color-defined red target. Under these conditions, the red color cues and the white onset cues would change their roles: When participants searched for a red color target, a red color cue would match the top-down control settings, and a white onset cue would not match the top-down control settings. This allowed us to unconfound the roles of colors and of top-down matching (vs. nonmatching) for the intertrial contingencies. If the intertrial contingencies of the validity effects in Experiments 1 and 2 were due to the use of red cues, we expected to find an intertrial contingency of the validity effect of the matching cues in Experiment 3 . Instead, if the intertrial contingencies in Experiments 1 and 2 were based on the lower task relevance of the nonmatching cues, we expected to see no such intertrial contingency of the validity effect of the red cues, because in Experiment 3 the red cue matched the control settings. In addition, if intertrial 
contingencies are generally found with nonmatching cues, we expected to observe an intertrial contingency with the white onset cues (here: the nonmatching cues).

\section{Method}

Participants A total of 22 (16 female, six male; $M_{\text {Age }}=$ 24.50 years, $S D_{A g e}=7.12$ ) new participants were tested. The ERs ranged from $0.26 \%$ to $13.73 \%$.

Procedure Participants searched for a red color target that was presented simultaneously with three white distractors at the remaining placeholders (see also Fig. 1, right panel). Most importantly, we used an SOA of $100 \mathrm{~ms}$, instead of $150 \mathrm{~ms}$ as in Experiment 1 and 2. In a pretest color-search experiment, we used the standard SOA of $150 \mathrm{~ms}$ but did not obtain any intertrial contingencies (see the Appendix). We think that color search is more difficult than onset search and that due to the higher RTs in the color-search conditions, we might have missed out on parts of a rather short-lived nonmatching validity effect. By shortening the SOA, we thus aimed at compensating for the RT delay. This increased the sensitivity of our method for nonmatching validity effects (see Liao \& Yeh, 2013) and their intertrial contingencies.

\section{Results}

Reaction times Trials following an error trial (6.33\%) and trials with RTs below $150 \mathrm{~ms}$ (additional $0.01 \%$ ) were removed from the analysis. Unless otherwise noted, all validity effects are significantly different from zero (all $p \mathrm{~s}<.05$ ). Table 1 (lower rows) provides a detailed overview of the RTs and ERs for Experiment 3.

The validity effect was larger in $n$-matching trials $(75 \mathrm{~ms})$ than in $n$-nonmatching trials (6 ms), $F(1,21)=232.40, p<$ .01 . In the $n$-nonmatching trials, the validity effect was not different from zero, $t(21)=1.62, p=.12$. Furthermore, the validity effect was larger after $n-1$ valid (45 ms) than after $n-1$ invalid (36 ms) trials, $F(1,21)=4.28, p=.05$, indicating an intertrial contingency of the validity effect. Importantly, this intertrial contingency was modulated by $n$-matching, $F(1,21)$ $=8.49, p=.01$. The validity effects in $n$-matching trials were not different following $n-1$ invalid (76 ms) and $n-1$ valid (74 ms) trials, $t(21)=0.32, p=.75$. Only in $n$-nonmatching trials was the validity effect larger following $n-1$ valid (16 ms) than $n-1$ invalid ( $-4 \mathrm{~ms}$ ) conditions. Note that the latter validity effect (in $n-1$ invalid and $n$-nonmatching trials) was not significantly different from zero, $t(21)=-1.27, p=.22$. No other effects were found, all nonsignificant $F_{\mathrm{S}}<3.13$, all $p \mathrm{~s}>$ .09. Figure 4 summarizes the results of Experiment 3.

Error rates The main effect of $n-1$ cue type came closest to significance, $F(1,21)=3.17, p=.09$, with a tendency toward a larger validity effect after $n-1$ nonmatching trials $(1.64 \%)$ than after $n-1$ matching trials $(0.84 \%)$. No other effects were found, all nonsignificant $F \mathrm{~s}<2.77$, all $p \mathrm{~s}>.11$.

\section{Discussion}

Experiment 3 showed that the intertrial contingencies of validity effects for nonmatching cues can also be found if participants search for color-defined targets. With a white nonmatching onset cue, the validity was stronger following $n-1$ valid than following $n-1$ invalid trials. With matching cues, no such intertrial contingencies were found. This resembled the findings of Experiments 1 and 2. However, we did not find an inverted validity effect of the $n$-nonmatching cues following an $n-1$ invalid trial. This could be explained in terms of suppression difficulty: The onset cue was of a higher luminance than the red color cue, and might therefore be harder to suppress.

\section{General discussion}

In the present study, we tested whether the attentional capture effects of matching cues, nonmatching cues, or both could be higher following $n-1$ valid trials than following $n-1$ invalid trials. Using the well-established contingent capture paradigm of Folk et al. (1992), we did find an intertrial contingency of attentional capture, in the form of a stronger validity effect following $n-1$ valid trials than following $n-1$ invalid trials. However, on closer inspection this intertrial contingency effect was restricted to $n$-nonmatching conditions.

This finding could be due to conflict adaptation or expectancies, but theoretically it could also point to supportive roles of position priming and maybe even cue-color repetitions for attentional capture by nonmatching cues (cf. Huang et al., 2004; Maljkovic \& Nakayama, 1996). However, in Experiment 2, in which cue and target positions were never repeated across trials, we still found an intertrial contingency of the validity effect for the nonmatching cues. Therefore, we can conclude that the intertrial contingency of attention capture depends neither on the learning of the exact target positions, nor on the repetition of the exact cue-target position relations, nor on position priming alone.

In addition, in the $n$-nonmatching conditions, a differently colored and attended-to target was presented in-between the cues in trials $n-1$ and $n$. Participants could have been slightly more inclined to redirect their attention to the nonmatching cue in trial $n$ if its color repeated from trial $n-1$ to $n$ (see Huang et al., 2004, for discussion of a possible principle). However, color priming per se would have been strongest in $n$-matching conditions, in which the color of the cue in trial $n$ was always a repetition of the color of the target in $n-1$. Yet, in Experiments $1-3$, we found no significant intertrial contingency of the 


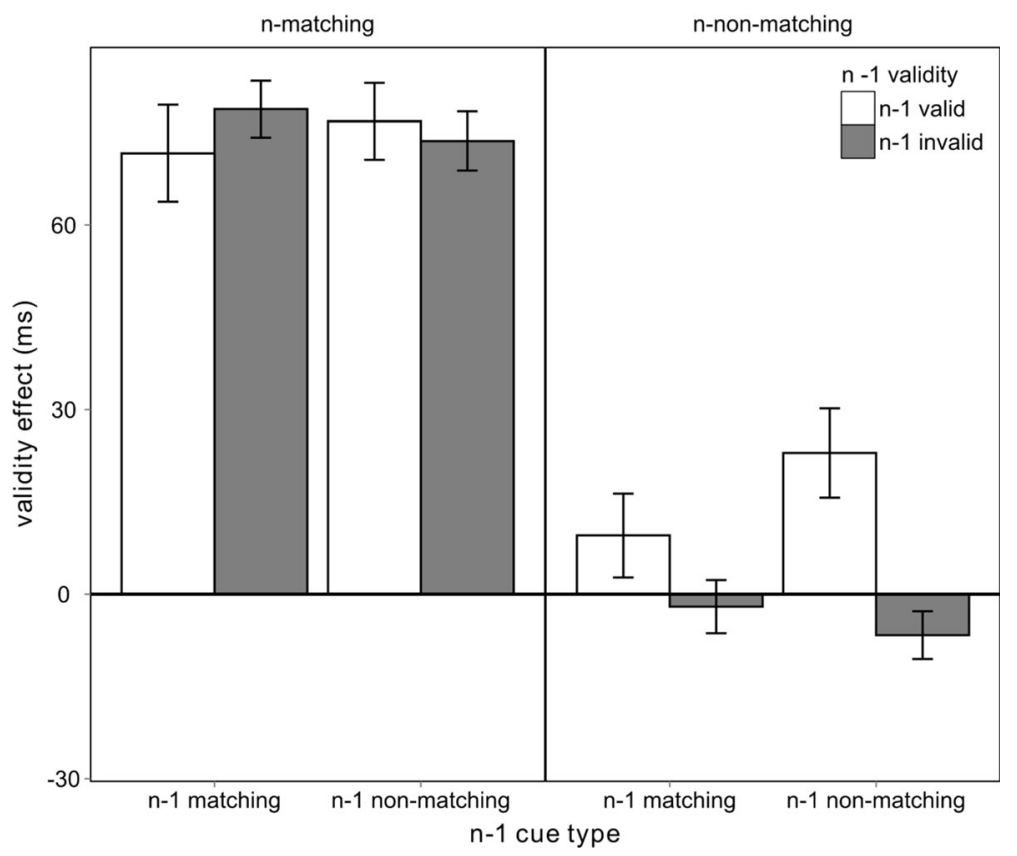

Fig. 4 Validity effects ( $n$-invalid RT $-n$-valid RT) as a function of $n$ cue type ( $n$-matching, left panel; $n$-nonmatching, right panel), $n-1$ validity ( $n-1$ valid, white bars; $n-1$ invalid, gray bars), and $n-1$ cue type ( $x$ -

validity effect with matching cues. Some authors have argued that stronger capture by matching than by nonmatching cues could be due to more intertrial priming of attention capture by the matching cues in trial $n$ through the similarly colored targets in trial $n-1$ (Awh et al., 2012; Theeuwes, 2013). Although a number of studies have confirmed that more capture by topdown matching cues than by nonmatching cues can be found with changes from an $n-1$ target color to an $n$ cue color (Ansorge \& Horstmann, 2007; Ansorge, Kiss, \& Eimer, 2009; Folk \& Remington, 2008; Worschech \& Ansorge, 2012; for a review, see Lamy \& Kristjánsson, 2013), in the original experimental protocol of Folk et al. (see also Folk \& Remington, 1998), participants searched for only one target color. This implies that all top-down matching cues were also potentially intertrial color primed by the preceding target. According to this line of argument, very strong intertrial priming of color could have prevented the influence of $n-1$ cuetarget validity on the validity effects in the matching conditions. For example, a very strong influence of intertrial color priming on attention might overcome a smaller effect based on expecting a helpful (after an $n-1$ valid trial) or less helpful (after an $n-1$ invalid trial) cue.

Instead, the intertrial contingency for the nonmatching cues must have reflected an influence such as the expectancy-based strategic use of cues after $n-1$ valid trials but not after $n-1$ invalid trials (Gratton et al., 1992), or more adaptation of control with the nonmatching cues (Botvinick et al., 2001). In line with active suppression (cf. Botvinick et al., 2001), we found inverted validity effects of the $n$-nonmatching cues after $n-1$ invalid trials in Experiments 1 and 2. If the $n-1$ axis) in Experiment 3 (color search). Error bars represent the standard errors of the means (SEMs)

nonmatching cues were harmful (i.e., invalid), they were actively suppressed in trial $n$. For example, in Experiments 1 and 2 , participants might have actively suppressed the red color cue from the start of a display. However, this strategy might have been more difficult with the higher-luminance nonmatching onset cues. In line with this argument, we found no inverted validity effect of the nonmatching onset cue following an $n-1$ invalid trial in Experiment 3.

This brings us to the limitations of the present study. Although we have selected a widely popular paradigm for our study and did not change its main characteristics, future studies would potentially benefit from doing so. For example, similar to Folk et al. (1992) we used a color red with a luminance that was lower than that of the white stimuli. Although it could be that participants relied on chromaticity to find the relevant red targets, it remains possible that the latencies of luminance processing also influence the time that it takes to find the target or to process the cue. Future studies could therefore use luminance-equated red and white colors or could parametrically vary the luminances of the cues to systematically investigate the influence of this variable on intertrial contingencies. Likewise, future studies could also use more than one target color to test whether different degrees of intertrial contingency could also be found for matching cues that are not primed versus cues that are primed by the color of the target in $n-1$.

\section{Conclusion}

The most interesting result of the present study was that nonmatching cues captured attention, provided that a valid 
cue had been used in the previous trial $n-1$. This finding holds true even though the cues were not predictive of the target position. The zero capture effects in nonmatching conditions could be the result of averaging across standard validity effects and inverted validity effects; both capture and suppression of capture could contribute to the performance in the nonmatching conditions. This finding also indicates that contingent-capture effects (defined as validity effect differences between matching and nonmatching cues) reflect more than capture by preexperimentally implemented search settings, and more than combined intertrial feature and position priming alone.

Author Note We thank Arnold Burghardt and David Modlinger for their help with the data collection. Supported by Wiener Wissenschaftsund Technologiefonds Grant No. CS11-009 to U.A., Shelley Buchinger, and Otmar Scherzer.

\section{Appendix}

This was our first experiment in which we tested for intertrial contingencies when participants had to search for a colordefined red target. We used the same procedure as in Experiment 1, with the difference that participants searched for a red color target that was concomitantly presented with three white distractors.

\section{Method}

Participants A group of 26 new participants (18 female, eight male; $M_{\text {Age }}=23.48$ years, $S D_{\text {Age }}=3.44$ ) were tested. One participant was excluded because of more than $20 \%$ error trials. The ERs of this sample ranged between $1.82 \%$ and $15.89 \%$

Procedure The procedure was the same as in Experiment 1. This time, we used one red color target that was presented with three white nontargets per target display, and the participants had to search for the red target and report its identity (see also Fig. 1, right panel, but note the exception that the duration of the fixation display between cue and target was $100 \mathrm{~ms}$, and not $50 \mathrm{~ms}$ as depicted).

\section{Results}

Reaction times All trials following an error trial (6.92\%), as well as all trials with RTs below $150 \mathrm{~ms}$ (an additional $0.09 \%$ ) were removed from the analysis. Mean correct RTs and ERs are listed in Table 2.

The analysis yielded a significant main effect of $n$-cue type, $F(1,24)=269.33, p<.01$. This pattern of results conforms to the predictions of the contingent capture view, suggesting that
Table 2 Summary of reaction times (RTs; in milliseconds) and error rates (ERs; in \%)

\begin{tabular}{|c|c|c|c|c|c|c|c|c|c|}
\hline \multirow[b]{4}{*}{ Trial $n-1$} & & \multicolumn{8}{|c|}{ Trial $n$} \\
\hline & & \multicolumn{4}{|c|}{ Matching Cue Type } & \multicolumn{4}{|c|}{ Nonmatching Cue Type } \\
\hline & & \multicolumn{2}{|c|}{ Valid } & \multicolumn{2}{|c|}{ Invalid } & \multicolumn{2}{|c|}{ Valid } & \multicolumn{2}{|c|}{ Invalid } \\
\hline & & RT & ER & RT & ER & $\mathrm{RT}$ & ER & RT & ER \\
\hline \multirow{2}{*}{$\begin{array}{l}\text { Matching cue } \\
\text { type }\end{array}$} & Valid & 500 & 5.07 & 563 & 8.60 & 527 & 4.60 & 529 & 4.07 \\
\hline & Invalid & 487 & 4.89 & 562 & 8.85 & 523 & 4.62 & 527 & 5.81 \\
\hline \multirow{2}{*}{$\begin{array}{c}\text { Nonmatching } \\
\text { cue type }\end{array}$} & Valid & 498 & 5.03 & 564 & 8.64 & 520 & 5.38 & 522 & 5.43 \\
\hline & Invalid & 489 & 5.91 & 567 & 8.81 & 526 & 4.80 & 523 & 5.21 \\
\hline
\end{tabular}

The table is split by the steps of the variables cue type (matching vs. nonmatching) and cue validity (valid vs. invalid) for both trials $n$ and $n-1$.

only $n$-matching cues led to a validity effect of $70 \mathrm{~ms}$, whereas $n$-nonmatching cues created no $n$ validity effect $[1 \mathrm{~ms} ; t$ test against zero: $t(24)=0.45, p=.66]$. Close to significance came an interaction between $n-1$ validity and $n$ cue type, $F(1,24)=$ $3.52, p=.07$. For $n$-matching trials, the validity effect was slightly larger after $n-1$ valid than $n-1$ invalid trials (76 vs. $64 \mathrm{~ms}), t(24)=-2.03, p=.05$. No such trend was found for $n$ nonmatching trials ( 0 vs. $2 \mathrm{~ms}$ ), $t(24)=0.45, p=.66$. No other significant effects were found, all $F_{\mathrm{s}}<2.69$, all $p \mathrm{~s}>.15$.

Error rates We found a main effect of $n$ cue type, $F(1,24)=$ $13.78, p<.01$. The validity effect was slightly larger in $n$ matching than in $n$-nonmatching trials (1.94\% vs. 1.64). The best evidence for a Gratton effect was a main effect of $n-1$ validity, $F(1,24)=5.11, p=.03$ : The validity effect was larger after $n-1$ valid than after $n-1$ invalid trials $(2.77 \%$ vs. $0.81 \%$ ). No other significant effects were found, all $F_{\mathrm{S}}<1.29$, all $p \mathrm{~s}>.27$.

\section{References}

Anderson, B. A., \& Folk, C. L. (2012). Dissociating location-specific inhibition and attention shifts: Evidence against the disengagement account of contingent capture. Attention, Perception, \& Psychophysics, 74, 1183-1198. doi:10.3758/s13414-012-0325-9

Ansorge, U., \& Horstmann, G. (2007). Preemptive control of attentional capture by color: Evidence from trial-by-trial analysis and ordering of onsets of capture effects in RT distributions. Quarterly Journal of Experimental Psychology, 60, 952-975.

Ansorge, U., Kiss, M., \& Eimer, M. (2009). Goal-driven attentional capture by invisible colors: Evidence from event-related potentials. Psychonomic Bulletin \& Review, 16, 648-653. doi:10.3758/PBR. 16.4.648

Awh, E., Belopolsky, A. V., \& Theeuwes, J. (2012). Top-down versus bottom-up attentional control: A failed theoretical dichotomy. Trends in Cognitive Sciences, 16, 437-443. doi:10.1016/j.tics. 2012.06.010 
Belopolsky, A. V., Schreij, D., \& Theeuwes, J. (2010). What is top-down about contingent capture? Attention, Perception, \& Psychophysics, 72, 326-341. doi:10.3758/APP.72.2.326

Botvinick, M. M., Braver, T. S., Barch, D. M., Carter, C. S., \& Cohen, J. D. (2001). Conflict monitoring and cognitive control. Psychological Review, 108, 624-652. doi:10.1037/0033-295X.108.3.624

Brainard, D. H. (1997). The psychophysics toolbox. Spatial Vision, 10, 433-436. doi:10.1163/156856897X00357

Carmel, T., \& Lamy, D. (2014). The same-location cost is unrelated to attentional settings: An object-updating account. Journal of Experimental Psychology: Human Perception and Performance, 40, $1465-1478$

Carter, C. S., \& van Veen, V. (2004). Anterior cingulate cortex and conflict detection: An update of theory and data. Cognitive, Affective, \& Behavioral Neuroscience, 7, 367-379. doi:10.3758/CABN.7.4.367

Eimer, M., \& Kiss, M. (2008). Involuntary attentional capture is determined by task set: Evidence from event-related brain potentials. Journal of Cognitive Neuroscience, 20, 1423-1433. doi:10.1162/ jocn.2008.20099

Folk, C. L., \& Remington, R. (1998). Selectivity in distraction by irrelevant featural singletons: Evidence for two forms of attentional capture. Journal of Experimental Psychology: Human Perception and Performance, 24, 847-858. doi:10.1037/0096-1523.24.3.847

Folk, C. L., \& Remington, R. W. (2008). Bottom-up priming of top-down attentional control settings. Visual Cognition, 16, 215-231. doi:10. 1080/13506280701458804

Folk, C. L., Remington, R. W., \& Johnston, J. C. (1992). Involuntary covert orienting is contingent on attentional control settings. Journal of Experimental Psychology: Human Perception and Performance, 18, 1030-1044. doi:10.1037/0096-1523.18.4.1030

Gratton, G., Coles, M. G., \& Donchin, E. (1992). Optimizing the use of information: Strategic control of activation of responses. Journal of Experimental Psychology: General, 121, 480-506. doi:10.1037/ 0096-3445.121.4.480

Hommel, B. (1998). Event files: Evidence for automatic integration of stimulus-response episodes. Visual Cognition, 5, 183-216. doi:10. 1080/713756773

Hommel, B., Proctor, R. W., \& Vu, K.-P. L. (2004). A feature-integration account of sequential effects in the Simon task. Psychological Research, 68, 1-17. doi:10.1007/s00426-003-0132-y

Huang, L., Holcombe, A. O., \& Pashler, H. (2004). Repetition priming in visual search: Episodic retrieval, not feature priming. Memory \& Cognition, 32, 12-20. doi:10.3758/BF03195816

Jongen, E. M. M., \& Smulders, T. Y. (2007). Sequence effects in a spatial cueing task: Endogenous orienting is sensitive to orienting in the preceding trial. Psychological Research, 71, 516-523.

Lamy, D. F., \& Kristjánsson, Á. (2013). Is goal-directed attentional guidance just intertrial priming? A review. Journal of Vision, 13(3), 14. doi: $10.1167 / 13.3 .14$
Liao, H.-I., \& Yeh, S.-L. (2013). Capturing attention is not that simple: Different mechanisms for stimulus-driven and contingent capture. Attention, Perception, \& Psychophysics, 75, 1703-1714. doi:10. 3758/s13414-013-0537-7

Maljkovic, V., \& Nakayama, K. (1994). Priming of pop-out: I. Role of features. Memory \& Cognition, 22, 657-672. doi:10.3758/ BF0320925

Maljkovic, V., \& Nakayama, K. (1996). Priming of pop-out: II. The role of position. Perception \& Psychophysics, 58, 977-991. doi:10.3758/ BF03206826

Mayr, U., Awh, E., \& Laurey, P. (2003). Conflict adaptation effects in the absence of executive control. Nature Neuroscience, 6, 450-452.

Mordkoff, J. T., Halterman, R., \& Chen, P. (2008). Why does the effect of short-SOA exogenous cuing on simple RT depend on the number of display locations. Psychonomic Bulletin \& Review, 15, 819-824. doi:10.3758/PBR.15.4.819

Müller, H. J., Geyer, T., Zehetleitner, M., \& Krummenacher, J. (2009). Attentional capture by salient color singleton distractors is modulated by top-down dimensional set. Journal of Experimental Psychology: Human Perception and Performance, 35, 1-16. doi: 10.1037/0096-1523.35.1.1

Posner, M. I. (1980). Orienting of attention. Quarterly Journal of Experimental Psychology, 32, 3-25. doi:10.1080/ 00335558008248231

Qian, Q., Shinomori, K., \& Song, M. (2012). Sequence effects by nonpredictive arrow cues. Psychological Research, 76, 253-262. doi: 10.1007/s00426-011-0339-2

Schlaghecken, F., \& Martini, P. (2012). Context, not conflict, drives cognitive control. Journal of Experimental Psychology: Human Perception and Performance, 38, 272-278.

Schmidt, J. R. (2013). Questioning conflict adaptation: Proportion congruent and Gratton effects reconsidered. Psychonomic Bulletin \& Review, 20, 615-630. doi:10.3758/s13423-012-0373-0

Theeuwes, J. (2010). Top-down and bottom-up control of visual selection. Acta Psychologica, 123, 77-99. doi:10.1016/j.actpsy.2010.02. 006

Theeuwes, J. (2013). Feature-based attention: It is all bottom-up priming. Philosophical Transactions of the Royal Society B, 368, 20130055. doi:10.1098/rstb.2013.0055

Theeuwes, J., Atchley, P., \& Kramer, A. F. (2000). On the time course of top-down and bottom-up control of visual attention. In S. Monsell \& J. Driver (Eds.), Control of cognitive processes: Attention and performance XVIII (pp. 105-125). Cambridge, MA: MIT Press.

Verguts, T., \& Notebaert, W. (2008). Hebbian learning of cognitive control: Dealing with specific and nonspecific adaptation. Psychological Review, 115, 518-525. doi:10.1037/0033-295X.115. 2.518

Worschech, F., \& Ansorge, U. (2012). Top-down search for color prevents voluntary directing of attention to informative singleton cues. Experimental Psychology, 59, 153-162. 